\title{
A case of oral cancer with preoperative transcatheter aortic valve implantation for aortic stenosis: A case report
}

\author{
TOMOKI KATO $^{1,2}$, AKIRA MATAYOSHI ${ }^{2}$ and TOSHIYUKI NAKASONE ${ }^{2}$ \\ ${ }^{1}$ Department of Oral and Maxillofacial Functional Rehabilitation, Graduate School of Medicine, University of The Ryukyus; \\ ${ }^{2}$ Department of Oral and Maxillofacial Surgery, University Hospital of The Ryukyus, Nishihara, Okinawa 903-0215, Japan
}

Received November 26, 2019; Accepted April 30, 2020

DOI: $10.3892 / \operatorname{mco} .2020 .2106$

\begin{abstract}
Aortic valve stenosis [or aortic stenosis, (AS)] is a risk factor in non-cardiac surgery. Guidelines from the European Heart Association recommended that AS treatment should be applied prior to non-cardiac surgery. Transcatheter aortic valve implantation (TAVI) is a method of minimally invasive AS treatment. The present study reports a case of tongue excision and neck dissection after TAVI for a patient with tongue cancer and AS. A 79-year-old woman was diagnosed with AS following preoperative examination for tongue cancer. The patient was judged as a high-risk case for open-heart surgery after consultation with staff at the University Hospital of The Ryukyus. It was concluded that TAVI should be performed prior to cancer surgery. On day 21 following TAVI, cancer surgery was performed and the patient was discharged from hospital 22 days after tongue cancer surgery. To the best of our knowledge, there is no report of oral cancer surgery after TAVI. TAVI is generally used as a short-term treatment strategy for AS associated with head and neck cancer due to its minimally invasive nature. In the present case, oral cancer surgery was performed closely following TAVI without serious complications. The case demonstrated that TAVI might be useful for severe AS patients with high surgical risk prior to cancer resection surgery. However, further cases are required to confirm the safety and effectiveness of TAVI treatment strategies.
\end{abstract}

Correspondence to: Dr Tomoki Kato, Department of Oral and Maxillofacial Functional Rehabilitation, Graduate School of Medicine, University of The Ryukyus, 207 Uehara, Nishihara, Okinawa 903-0215, Japan

E-mail: t.kato.clpc@gmail.com

Abbreviations: AS, aortic valve stenosis; SAVR, surgical aortic valve replacement; TAVI, transcatheter aortic valve implantation; FDG-PET, 2-[18F]-fluoro-2-deoxy-D-glucose-positron emission tomography

Key words: head and neck cancer, tongue cancer, transcatheter aortic valve implantation, aortic valve stenosis

\section{Introduction}

As the population ages, the incidence of head and neck cancer in older people has increased. Aged patients will have many complications, but aortic valve stenosis [or aortic stenosis, (AS)] may be found in preoperative examination for non-cardiac surgery such as cancer surgery.

The European Heart Association guidelines recommend surgical aortic replacement (SAVR) for patients with severe symptomatic AS before non-cardiac surgery. SAVR is recommended for patients with severe asymptomatic AS only when the risk of non-cardiac surgery is high and when the risk of SAVR is low (1).

Therefore, cancer patients who are contraindicated for SAVR may not be able to receive sufficient cancer treatment. Even if SAVR can be performed, the burden of surgery may be great, and the timing of cancer-bearing surgery may be delayed.

In this study, we performed low-invasive transcatheter aortic valve implantation (TAVI) before carrying out oral cancer surgery at an early stage for a patient with advanced AS and tongue cancer. To our knowledge, there are no reports of oral cancer surgery after TAVI. Therefore, we also provide a summary and literature review to help evaluate the safety and efficacy of oral cancer surgery after TAVI.

\section{Case report}

The patient was a 79-year-old woman who had a history of hypertension, diabetes, angina, cerebral infarction, and hysteromyoma after artificial joint replacement. Her family history was unremarkable. Around January 2019, a mass was noticed on the left tongue margin, but it was judged to be stomatitis and left untreated. In May 2019, she visited a dental clinic for denture fabrication. She was subsequently referred to our department because of the suspicion of tongue cancer. The patient's physique was moderate and her nutritional status was good. Her face was symmetrical, and several elastic, mobile, bean-sized lymph nodes were palpated. No tenderness was observed in the submandibular region. However, left lower body paralysis due to cerebral infarction was observed.

Intraoral findings showed a mass consolidation measuring approximately $25 \times 15 \mathrm{~mm}$ with no spontaneous pain or tenderness in the left tongue margin (Fig. 1). Hemorrhage, ulceration, 


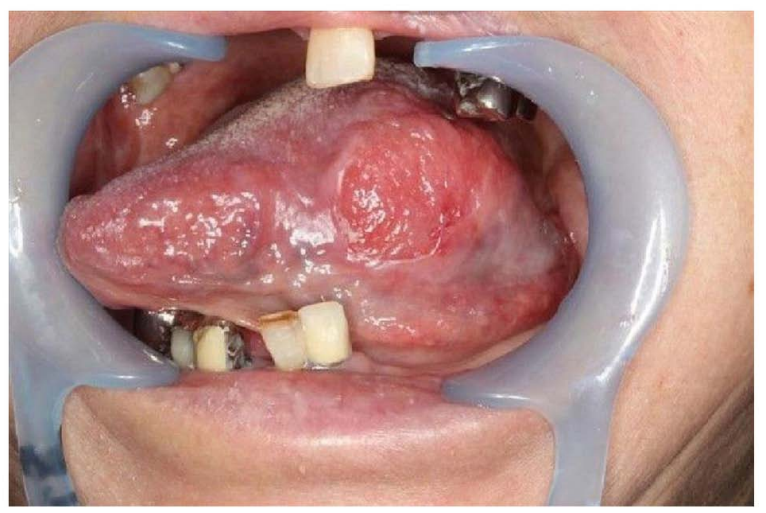

Figure 1. Clinical photograph of the left tongue tumor lesion during initial oral examination. Physical examination revealed an elastic, hard mass on the left side of the tongue measuring $25 \times 15 \mathrm{~mm}$.
A

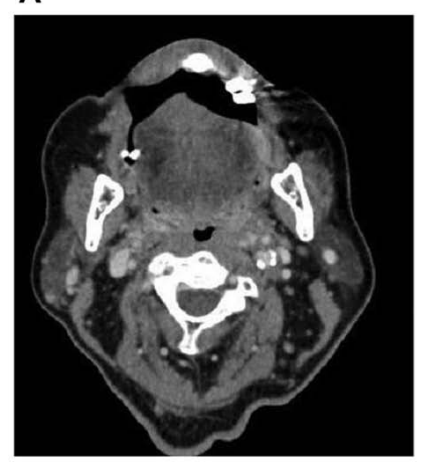

\section{B}

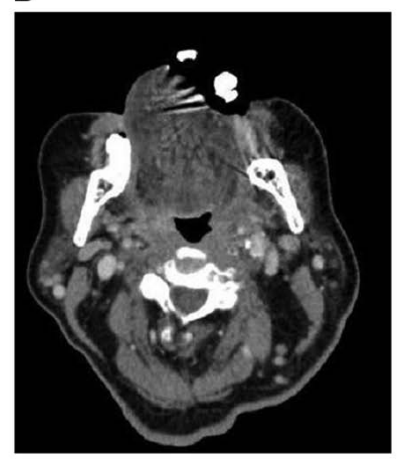

Figure 2. Contrast-enhanced computed tomography images of axial sections. (A) Contrast-enhanced computed tomography revealed a tumor with an unclear border exhibiting a contrast effect on the left lingual margin. (B) Contrast-enhanced computed tomography revealed an enlarged, uniformly circular lymph node in the upper left internal deep cervical lymph node.
A

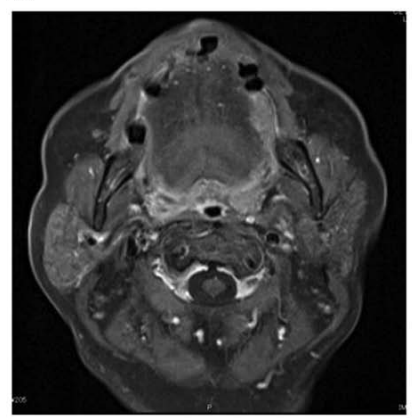

B

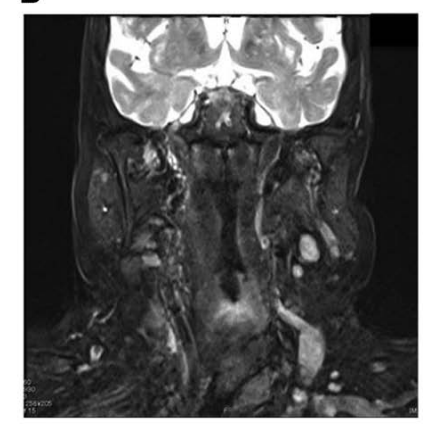

Figure 3. MRI images. (A) Axial sections. (B) Coronal sections. (A) Contrast-enhanced MRI revealing a high-signal region with a uniform internal contrast on the left side of the tongue. (B) Contrast-enhanced MRI exhibiting a high-signal region in the left upper internal deep cervical lymph node.

tongue movement disorder, and sensory disturbance were not observed.

Contrast-enhanced computed tomography revealed a tumor with an unclear border showing a contrast effect and measuring 10x23 mm on the left lingual margin (Fig. 2A).

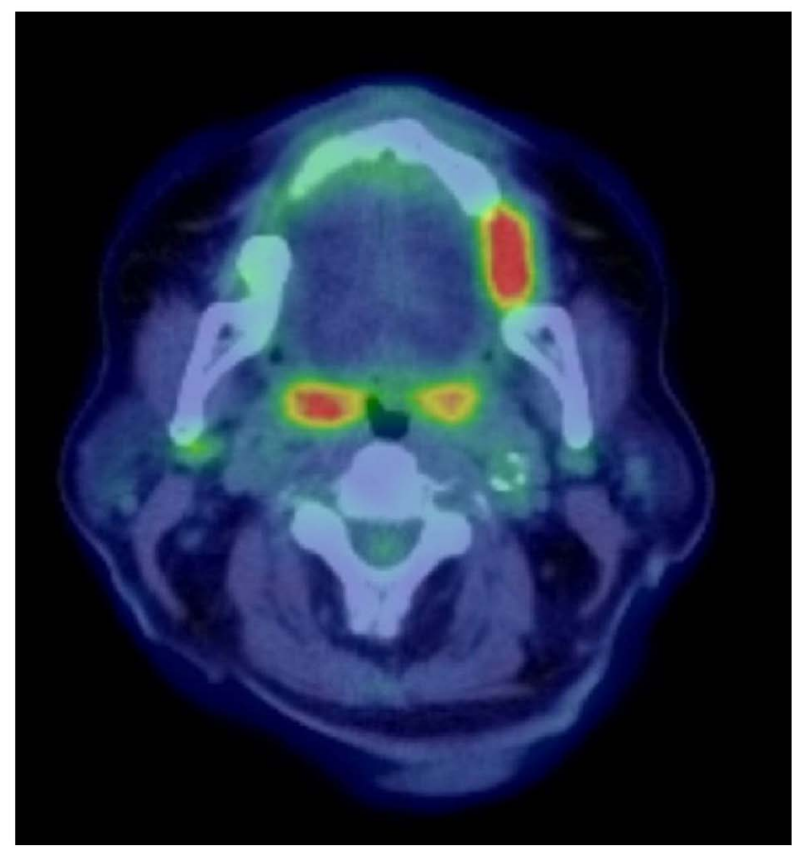

Figure 4. FDG-PET/CT revealing abnormal FDG accumulation in the left lingual margin (SUVmax, 9.14) and bilateral superior internal cervical lymph nodes (right: SUVmax, 2.65; left: SUVmax, 3.93). FDG-PET/CT, 2 -[ $\left.{ }^{18} \mathrm{~F}\right]$-fluoro-2-deoxy-D-glucose positron emission tomography/computed tomography.

The left upper internal deep cervical lymph node showed an enlarged, uniformly circular lymph node measuring 9x15 mm (Fig. 2B). Contrast-enhanced MRI showed a high-signal region with a uniform internal contrast area measuring 10x23 mm on the left side of the tongue (Fig. 3A) and a high-signal region measuring $9 \times 15 \mathrm{~mm}$ in the left upper internal deep cervical lymph node (Fig. 3B). Preoperative use of $2-\left[{ }^{18} \mathrm{~F}\right]-$ fluoro-2-deoxy-D-glucose positron emission tomography/ computed tomography (FDG-PET/CT) showed abnormal FDG accumulation in the left lingual margin (SUVmax: 9.14) and bilateral superior internal cervical lymph nodes (right: SUVmax: 2.65, left: SUVmax: 3.93) (Fig. 4). Biopsy findings indicated squamous cell carcinoma (Fig. 5). Ultrasonography showed that the internal structure of the lymph node was uniform and the morphology of the lymph node was round. The lymph nodes examined by palpation were mobile and not indurated. Under the diagnosis of tongue cancer (cT2NOM0), partial tongue excision and neck dissection were scheduled. We thus staged the tumor as N0 clinically by comprehensively physical assessments and various imaging findings. However, the possibility of metastasis could not be ruled out, and it was anticipated that postoperative follow-up would be difficult because the patient lives on a remote island. Therefore, we performed preventive neck dissection because the detection of cervical metastases could be delayed.

In May 2019, a biopsy was performed under local anesthesia. Biopsy findings at this time indicated squamous cell carcinoma. Therefore, partial tongue excision and left functional neck dissection were scheduled under general anesthesia, and a preoperative examination was performed. AS was confirmed by echocardiography. Cardiac ultrasonography revealed an aortic valve maximum blood flow of $5.0 \mathrm{~m} / \mathrm{s}$, aortic valve mean 


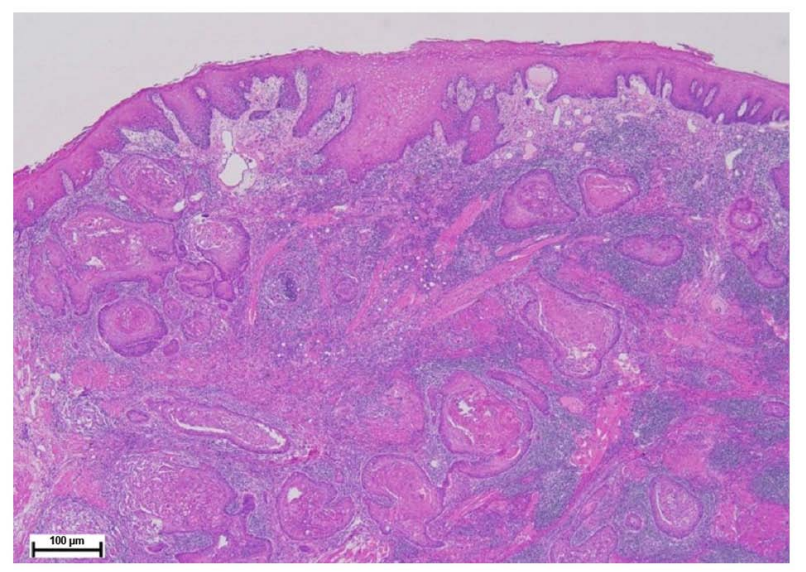

Figure 5. Histopathological examination revealing squamous cell carcinoma. The tumor specimen exhibited moderate proliferation of squamous cell carcinoma cells (staining, hematoxylin and eosin; magnification, x20).

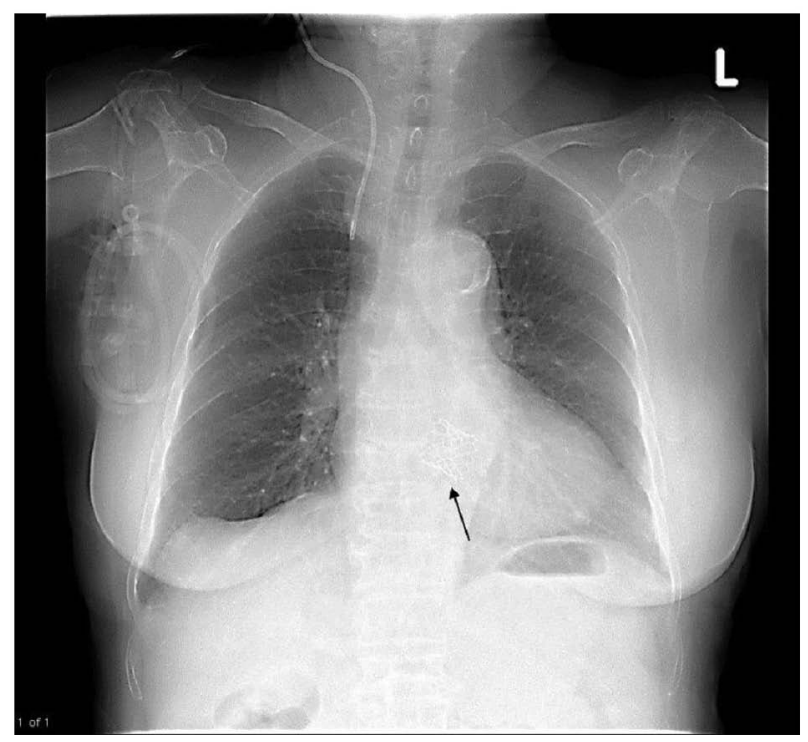

Figure 6. Chest X-ray after transcatheter aortic valve implantation Implantation of a $23 \mathrm{~mm}$ SAPIEN 3 valve (arrow) is observed. L, left hand side.

pressure difference of $58 \mathrm{mmHg}$, aortic valve opening area of $0.5 \mathrm{~cm}^{2}$, severe AS, and left ventricular wall thickening. EF of $63.4 \%$ and left ventricular wall motion were within normal limits. Based on these findings, AS treatment was considered to be necessary before tongue cancer surgery. However, after consultation with the heart team, the patient was judged to be a high-risk case for open-heart surgery according to her advanced age and history of diabetes, cerebral infarction, and angina. We therefore decided to perform TAVI with the femoral artery approach.

Based on findings from coronary computed tomography angiography during preoperative examination of TAVI, we suspected coronary artery stenosis, but coronary blood flow was maintained by coronary angiography. In July 2019, a guide wire was passed from the left femoral artery to the aortic valve, and a 23 mm SAPIEN 3 valve (Edwards Lifesciences Corp.) was placed under rapid pacing (180 times/min) (Fig. 6). Only a small amount of periarticular regurgitation was present, and the coronary artery was well visualized. The patient was extubated in the operating room and left the bed the next day. Blood tests confirmed improvement of inflammatory responses and no platelet count decrease, and partial tongue excision, free skin grafting, and neck dissection were performed under general anesthesia 21 days after TAVI surgery. Histopathological findings showed that tissue was squamous cell carcinoma with prominent keratinization proliferated in solid alveolar form. Keratin pearls were occasionally found and nuclear atypia was relatively mild. The tumor follicle measured 30x22x12 mm and the depth of penetration was $11 \mathrm{~mm}$. There were no metastases in cervical lymph nodes. The patient was discharged without complications 22 days after tongue cancer surgery.

\section{Discussion}

AS is a narrowing of the opening of the aortic valve, leading to obstruction of left ventricular outflow, subsequent left ventricular systolic dysfunction, and ultimately long-term heart failure. In patients undergoing non-cardiac surgery, severe AS is a known high-risk factor for mortality and morbidity because it reduces coronary blood flow due to surgical stress and hemodynamic changes. The European Heart Association guidelines recommend SAVR for patients with severe symptomatic AS before non-cardiac surgery. However, for patients with severe asymptomatic AS, SAVR is recommended only when the risk of SAVR is low and when the risk of non-cardiac surgery is high (1). The severity and symptoms of AS, which may increase the risk of oral cancer surgery in such older cancer patients, may not be readily apparent. We prioritized TAVI for AS treatment in the present case because of her older age and multiple complications, which were contraindications for major invasive procedures such as thoracotomy, extracorporeal circulation, and cardiac arrest (2).

TAVI is a minimally invasive approach to replace an aortic valve that is different from SAVR in that it does not require cardiac arrest, extracorporeal circulation, and thoracotomy. In high-risk or ineligible patients, PARTNER trials have shown that treatment outcomes such as mortality, LV recovery, and morbidity after TAVI are comparable or superior to conventional treatment (3-5). Thus, it has been reported that TAVI can be a standard alternative treatment for high-risk surgical patients. In addition, certain benefits of TAVI have been reported in cancer patients because it does not require cardiopulmonary bypass. These include reducing the risk associated with tumor bleeding with anticoagulant disorders and administration of anticoagulants, and reducing tumor dissemination with immunosuppressive and inflammatory effects of cardiopulmonary bypass $(6,7)$. In severe AS patients in need of cancer treatment, TAVI may not only allow a smooth transition to the next treatment strategy due to minimal invasiveness, but also yield oncological benefits.

As far as we are aware, there have been no reports of TAVI being performed before surgery for head and neck cancers. There were a few reports found of TAVI procedures performed before surgery for cancers in other parts of the body (8-12). The interval between AS treatment and cancer treatment should be short to prevent malignant tumor progression. However, when SAVR and cancer-bearing surgery are performed in two 
stages, a short interval between operations may not be possible because of surgical tolerance. In addition, surgery decreases the body's natural defenses; thus, insufficient time between operations increases the risk of postoperative infection. To our knowledge, there is no report on the appropriate interval between TAVI and surgery, however, an interval of about 2-3 weeks between cancer resection surgery and TAVI was applied in colon cancer and lung cancer patients (8-12). We scheduled a similar time interval in the present case.

Due to factors such as age, heart failure, and cancer, 30-50\% of patients with symptomatic AS are considered ineligible for SAVR (3-5). Additionally, Bach et al reported that almost the same number of patients with SAVR had refused surgery (13). Previously, older cancer patients with severe AS, such as the present case, may not have been able to undergo severe AS or cancer treatment. However, TAVI is potentially useful for such patients. While there are several reports of cancer surgery after TAVI (8-12), to our knowledge, this is the first report of TAVI for a patient undergoing oral cancer surgery. Moreover, there is little data on the feasibility of TAVI for severe AS patients scheduled for cancer surgery and the impact on subsequent surgery. A greater accumulation of similar cases and further investigation of multiple factors, such as the appropriate treatment interval between TAVI and cancer surgery, perioperative antiplatelet drugs, and surgical indications, are needed.

In conclusion, we performed oral cancer surgery early after TAVI without serious complications. TAVI could be a useful option for severe AS patients with high surgical risk prior to cancer resection surgery. However, a greater accumulation of cases is needed to confirm the safety and effectiveness of TAVI-containing treatment strategies.

\section{Acknowledgements}

The authors would like to thank Dr Takaaki Nagano and Dr Shotaro Higa (both, Department of Thoracic and Cardiovascular Surgery, Graduate School of Medicine, University of the Ryukyus, Okinawa, Japan) and Dr Masashi Iwabuchi and Dr Hidekazu Ikemiyagi, (Department of Cardiovascular Medicine, Nephrology and Neurology, University of the Ryukyus, Okinawa, Japan) who contributed to patient treatment and care.

\section{Funding}

No funding was received.

\section{Availability of data and materials}

All data generated or analyzed during this study are included in this published article.

\section{Authors' contributions}

TK acquired patient data, performed the literature review and edited the manuscript. TN conceived and designed the present study. TN acquired the data, provided clinical advice and revised the manuscript. AM and TN evaluated specimens and gave histopathological advice. TK wrote the manuscript. All authors read and approved the final manuscript.

\section{Ethics approval and consent to participate}

The present report was submitted for ethical review to the Ethics Committee of the University of the Ryukyus (Okinawa, Japan), which waived the requirement for review per institutional protocol due to the study not containing content that requires ethical approval. The Ethics Committee approved the submission and publication of the manuscript. Written informed consent was obtained from the patient for the publication of this case report and accompanying images.

\section{Patient consent for publication}

Written informed consent was obtained from the patient for the publication of this case report and the accompanying images.

\section{Competing interests}

The authors declare that they have no competing interests.

\section{References}

1. Kristensen SD, Knuuti J, Saraste A, Anker S, Bøtker HE, Hert SD, Ford I, Gonzalez-Juanatey JR, Gorenek B, Heyndrickx GR, et al: 2014 ESC/ESA guidelines on non-cardiac surgery: Cardiovascular assessment and management: The joint task force on non-cardiac surgery: Cardiovascular assessment and management of the European Society of Cardiology (ESC) and the European Society of Anaesthesiology (ESA). Eur Heart J 35: 2383-2431, 2014.

2. Cribier A, Eltchaninoff $\mathrm{H}$, Bash A, Borenstein N, Tron $\mathrm{C}$, Bauer F, Derumeaux G, Anselme F, Laborde F and Leon MB: Percutaneous transcatheter implantation of an aortic valve prosthesis for calcific aortic stenosis: First human case description. Circulation 106: 3006-3008, 2002.

3. Elmariah S, Palacios IF, McAndrew T, Hueter I, Inglessis I, Baker JN, Kodali S, Leon MB, Svensson L, Pibarot P, et al: Outcomes of transcatheter and surgical aortic valve replacement in high-risk patients with aortic stenosis and left ventricular dysfunction: Results from the Placement of Aortic Transcatheter Valves (PARTNER) trial (cohort A). Circ Cardiovasc Interv 6: 604-614, 2013.

4. Mack MJ,Leon MB, Smith CR, Miller DC, Moses JW, Tuzcu EM, Webb JG, Douglas PS, Anderson WN, Blackstone EH, et al: 5-year outcomes of transcatheter aortic valve replacement or surgical aortic valve replacement for high surgical risk patients with aortic stenosis (PARTNER1): A randomised controlled trial. Lancet 385: 2477-2484, 2015.

5. Kapadia SR, Leon MB, Makkar RR, Tuzcu EM, Svensson LG, Kodali S, Webb JG, Mack MJ, Douglas PS, Thourani VH, et al: 5 -year outcomes of transcatheter aortic valve replacement compared with standard treatment for patients with inoperable aortic stenosis (PARTNER 1): A randomised controlled trial. Lancet 385: 2485-2491, 2015.

6. Scott BH, Seifert FC, Glass PS and Grimson R: Blood use in patients undergoing coronary artery bypass surgery: Impact of cardiopulmonary bypass pump, hematocrit, gender, age, and body weight. Anesth Analg 97: 958-963, 2003.

7. Pinto CA, Marcella S, August DA, Holland B, Kostis JB and Demissie K: Cardiopulmonary bypass has a modest association with cancer progression: A retrospective cohort study. BMC Cancer 13: 519, 2013.

8. Tamai K, Okamura S, Kitahara T, Minoji T, Takabatake H, Watanabe N, Yamamura N, Fukuchi N, Ebisui C, Yokouchi H, et al: Laparoscopic colectomy after transcatheter aortic valve implantation in an elderly patient with obstructive descending colon cancer and severe aortic stenosis: A case report. Surg Case Rep 5: 102, 2019.

9. Sakai T, Yahagi K, Miura S, Hoshino T, Yokota T, Tanabe K and Ikeda S: Transcatheter aortic valve implantation for patients with lung cancer and aortic valve stenosis. J Thorac Dis 10: E387-E390, 2018. 
10. Tanaka T, Yahagi K, Okuno T, Horiuchi Y, Kusuhara T, Yokozuka M, Miura S, Tanabe K, Miura S, Tanabe K and Tanabe K: Transcatheter aortic valve implantation in a patient with severe aortic valve stenosis, colon cancer, and obstructive ileus: A case report. J Cardiol Cases 17: 163-166, 2018.

11. Okuno T, Yahagi K, Horiuchi Y, Sato Y, Tanaka T, Koseki K, Osanai A, Yokozuka M, Miura S and Tanabe K: The role of transcatheter aortic valve replacement in the patients with severe aortic stenosis requiring major non-cardiac surgery. Cardiovasc Interv Ther 13: 345-351, 2019.
12. Nagata H, Kanzaki R, Kanou T, Ose N, Funaki S, Shintani Y, Minami M, Mizote I, Sakata Y, Maeda K, et al: Two cases of lobectomy for lung cancer after transcatheter aortic valve implantation. Surg Case Rep 4: 139, 2018.

13. Bach DS, Cimino N and Deeb GM: Unopoerated patients with severe aortic stenosis. J Am Coll Cardiol 50: 2018-2019, 2007.

(C) () $९$ This work is licensed under a Creative Commons cc) International (CC BY-NC-ND 4.0) License. 